\title{
Saúde mental e intervenções psicológicas diante da pandemia do novo coronavírus (COVID-19)
}

\author{
Mental health and psychological interventions during \\ the new coronavirus pandemic (COVID-19)
}

\author{
Beatriz SCHMIDT ${ }^{1}$ ID 0000-0003-2907-2297 \\ Maria Aparecida CREPALDI ${ }^{2}$ (D) 0000-0002-5892-7330 \\ Simone Dill Azeredo BOLZE2 (iD) 0000-0002-8715-8913 \\ Lucas NEIVA-SILVA1 (ID) 0000-0002-7526-2238 \\ Lauro Miranda DEMENECH ${ }^{3}$ (D) 0000-0002-7285-2566
}

\section{Resumo}

A pandemia do novo coronavírus (COVID-19) é a maior emergência de saúde pública que a comunidade internacional enfrenta em décadas. Além das preocupações quanto à saúde física, traz também preocupações quanto ao sofrimento psicológico que pode ser experienciado pela população geral e pelos profissionais da saúde envolvidos. O objetivo do presente estudo foi sistematizar conhecimentos sobre implicações na saúde mental e intervenções psicológicas diante da pandemia do novo coronavírus. Realizou-se revisão da literatura técnico-científica produzida em diferentes países, na perspectiva de sumarizar desenvolvimentos recentes ligados à COVID-19. Apresentam-se resultados sobre implicações da pandemia na saúde mental, identificação de grupos prioritários e orientações sobre intervenções psicológicas, considerando particularidades da população geral e dos profissionais da saúde. Por fim, discutem-se potencialidades e desafios para a prática dos psicólogos no contexto brasileiro durante a pandemia.

Palavras-chave: Coronavírus; Pandemias; Psicologia; Saúde mental.

$\checkmark v \nabla$

1 Universidade Federal do Rio Grande, Instituto de Ciências Humanas e da Informação, Programa de Pós-Graduação em Psicologia. Av. Itália, km 8, Bairro Carreiros, 96203-900, Rio Grande, RS, Brasil. Correspondência para/Correspondence to: B. SCHMIDT. E-mail:<psi.beatriz@gmail.com>.

2 Universidade Federal de Santa Catarina, Centro de Filosofia e Ciências Humanas, Programa de Pós-Graduação em Psicologia. Florianópolis, SC, Brasil.

3 Universidade Federal do Rio Grande, Faculdade de Medicina, Programa de Pós-Graduação em Ciências da Saúde. Rio Grande, RS, Brasil.

$\boldsymbol{\nabla} \mathbf{v}$

Como citar este artigo/How to cite this article

Schmidt, B., Crepaldi, M. A., Bolze, S. D. A., Neiva-Silva, L., \& Demenech, L. M. (2020). Saúde mental e intervenções psicológicas diante da pandemia do novo coronavírus (COVID-19). Estudos de Psicologia (Campinas), 37, e200063. http://dx.doi.org/10.1590/1982-0 $275202037 \mathrm{e} 200063$ 


\begin{abstract}
The new coronavirus pandemic (COVID-19) is the greatest public health emergency facing the international community in decades. Aside from the concerns related to physical health, it also brings up concerns related to the psychological distress that the general population and health professionals may experience. The aim of the current study was to systematize knowledge on the impacts of the new coronavirus pandemic on mental health, as well as on psychological interventions related to the disease. A review of the technical-scientific literature from different countries was carried out in order to summarize recent developments linked to the COVID-19. The results presented refer to the impacts of the pandemic on mental health, identification of priority groups, and guidance on psychological interventions, also considering the particularities of the general population and health professionals. Finally, we discuss both the potential and the challenges of practicing psychology in Brazil during the pandemic.
\end{abstract}

Keywords: Coronavirus; Pandemics; Psychology; Mental health.

O primeiro caso de infecção pelo novo coronavírus (Severe Acute Respiratory Syndrome Coronavirus $2^{4}$ - Sars-Cov-2) foi reportado na China, no início de dezembro de 2019 (Wang et al., 2020; Xiao, 2020). A rápida escalada da doença (Coronavirus Disease 2019 - COVID-19), com disseminação em nível global, fez com que a World Health Organization a considerasse uma pandemia. Em 16 de abril de 2020, o número de casos confirmados mundialmente superava dois milhões, ao passo que o número de mortes superava 130 mil (World Health Organization [WHO], 2020a). Nessa mesma data, o Brasil contava com 30.425 casos confirmados e 1.924 mortes (Ministério da Saúde, 2020a). Entretanto, estima-se que esses números sejam ainda maiores, dado que não levam em conta atrasos nas notificações ou casos positivos não testados (Russell et al., 2020). Estatísticas sugerem que o número de reprodução da COVID-19 (i.e., o número médio de novos casos gerados a partir de um caso) varia de 1,4 a 3,9 em diferentes localidades (Villela, 2020). Assim, o tempo de duração e os desdobramentos da pandemia ainda permanecem imprevisíveis (Xiao, 2020).

Até 16 de abril de 2020, quando a redação do presente artigo foi concluída, a COVID-19 já trazia um profundo impacto global, sendo considerada a síndrome respiratória viral mais severa desde a pandemia de influenza H1N1, em 1918 (Ferguson et al., 2020). As estimativas são de que essa pandemia, também conhecida como "gripe espanhola", levou a óbito entre vinte e cinquenta milhões de pessoas em todo o mundo, há pouco mais de cem anos (Matos, 2018). Os sintomas físicos da COVID-19 frequentemente envolvem tosse, febre e dificuldades respiratórias (Carvalho, Moreira, Oliveira, Landim, \& Rolim Neto, 2020) que podem levar à morte (Li et al., 2020b). O significativo número de casos que demandam internação hospitalar (Duan \& Zhu, 2020), incluindo cuidados em unidade de terapia intensiva, bem como a ausência de intervenções farmacológicas eficazes e seguras, tais como medicamentos ou vacinas, têm gerado preocupações quanto ao colapso do sistema de saúde em diferentes nações (Ferguson et al., 2020). Com o objetivo de reduzir os impactos da pandemia, diminuindo o pico de incidência e o número de mortes, alguns países têm adotado medidas tais quais isolamento de casos suspeitos, fechamento de escolas e universidades, distanciamento social de idosos e outros grupos de risco, bem como quarentena de toda a população (Brooks et al., 2020; Ferguson et al., 2020). Estima-se que essas medidas tendam a "achatar a curva" de infecção, ao favorecer um menor pico de incidência em um dado período, reduzindo as chances de que a capacidade de leitos hospitalares, respiradores e outros suprimentos seja insuficiente frente ao aumento repentino da demanda, o que se associaria a uma maior mortalidade (Ferguson et al., 2020).

Em linhas gerais, na vigência de pandemias, a saúde física das pessoas e o combate ao agente patogênico são os focos primários de atenção de gestores e profissionais da saúde, de modo que as implicações

"Tradução livre para a língua portuguesa: "Síndrome Respiratória Aguda Grave do Coronavírus 2". 
sobre a saúde mental tendem a ser negligenciadas ou subestimadas (Ornell, Schuch, Sordi, \& Kessler, 2020). Contudo, medidas adotadas para reduzir as implicações psicológicas da pandemia não podem ser desprezadas neste momento (Brooks et al., 2020; Xiao, 2020). Se isso ocorre, geram-se lacunas importantes no enfrentamento dos desdobramentos negativos associados à doença, o que não é desejável, sobretudo porque as implicações psicológicas podem ser mais duradouras e prevalentes que o próprio acometimento pela COVID-19, com ressonância em diferentes setores da sociedade (Ornell et al., 2020).

Estudos têm sugerido que o medo de ser infectado por um vírus potencialmente fatal, de rápida disseminação, cujas origens, natureza e curso ainda são pouco conhecidos, acaba por afetar o bem-estar psicológico de muitas pessoas (Asmundson \& Taylor, 2020; Carvalho et al., 2020). Sintomas de depressão, ansiedade e estresse diante da pandemia têm sido identificados na população geral (Wang et al., 2020) e, em particular, nos profissionais da saúde (Zhang et al., 2020a). Ademais, casos de suicídio potencialmente ligados às implicações psicológicas da COVID-19 também já foram reportados em alguns países como Coreia do Sul (Jung \& Jun, 2020) e Índia (Goyal, Chauhan, Chhikara, Gupta, \& Singh, 2020).

Afora as implicações psicológicas diretamente relacionadas à COVID-19, medidas para contenção da pandemia também podem consistir em fatores de risco à saúde mental. Em revisão de literatura sobre a quarentena, Brooks et al. (2020) identificaram que os efeitos negativos dessa medida incluem sintomas de estresse pós-traumático, confusão e raiva. Preocupações com a escassez de suprimentos e as perdas financeiras também acarretam prejuízos ao bem-estar psicológico (Shojaei \& Masoumi, 2020). Nessa conjuntura, tende ainda a aumentar o estigma social e os comportamentos discriminatórios contra alguns grupos específicos, como é o caso dos chineses, população primeiramente afetada pela infecção pelo novo coronavírus (Shimizu, 2020), bem como dos idosos, pois é nessa faixa etária que tem ocorrido o maior número de óbitos em decorrência da COVID-19 (Ornell et al., 2020).

Analisados em conjunto, todos esses fatores remetem à relevância de intervenções psicológicas alinhadas às necessidades emergentes no atual contexto de pandemia. Nas últimas semanas, foram publicados estudos narrando práticas exitosas que vêm sendo adotadas, especialmente no continente asiático (Duan \& Zhu, 2020; Jiang et al., 2020; Xiao, 2020; Zhou, 2020), orientações de associações e conselhos de Psicologia em diferentes países, como no Brasil (Conselho Federal de Psicologia [CFP], 2020a; 2020b), na Espanha (Consejo General de la Psicología da España, 2020), nos Estados Unidos (American Psychological Association, 2020), bem como recomendações para cuidados em saúde mental pela Organização Mundial da Saúde (WHO, 2020b). Adicionalmente, em 31 de março de 2020, foi publicada a Portaria n 639, do Ministério da Saúde, que dispõe sobre a ação estratégica "O Brasil Conta Comigo - Profissionais da Saúde", sobre a capacitação e o cadastramento de profissionais da saúde para o enfrentamento à COVID-19, incluindo psicólogos (Ministério da Saúde, 2020b).

Considerando o exposto, o objetivo do presente estudo é sistematizar conhecimentos sobre implicações na saúde mental e intervenções psicológicas diante da pandemia do novo coronavírus. Para tanto, realizou-se revisão narrativa da literatura, modalidade que permite a sumarização de estudos baseados em diferentes abordagens metodológicas sobre uma mesma temática, com apresentação descritiva dos achados (Pautasso, 2020). A revisão narrativa da literatura pode favorecer reflexões sobre a pandemia do novo coronavírus, por se tratar de problemática emergente, que demanda resposta rápida e efetiva - e que tem sido dada por cientistas e profissionais da saúde em todo o mundo, cujos esforços têm gerado novas publicações diariamente. Nesse sentido, a busca por materiais ocorreu por meio de sucessivas consultas a bases de dados e portais de pesquisa (Science Direct, PubMed, Scientific Electronic Library Online e Google Scholar), em diferentes dias do mês de março de 2020, com uso de descritores como "coronavirus", "COVID-19", "mental health", "psychological impact" e "psychological interventions". Também foram pesquisados sites de organizações ligadas à área da saúde e à Psicologia, em diferentes países, na perspectiva de buscar os conhecimentos mais recentes ligados à COVID-19. 
Além de descrever as experiências de profissionais da saúde mental em outras sociedades diante da pandemia do novo coronavírus, buscou-se refletir sobre possíveis aplicações dessas experiências no contexto nacional, dadas as particularidades da população brasileira. Para tanto, tomou-se como base a experiência dos autores, tanto no âmbito profissional quanto no de pesquisa desenvolvida nas áreas de Psicologia da Saúde e Psicologia Clínica em duas universidades de diferentes estados no Brasil - a Universidade Federal do Rio Grande e a Universidade Federal de Santa Catarina. Os achados dessa revisão narrativa são apresentados por meio de duas seções: "Implicações na saúde mental em decorrência da pandemia do novo coronavírus" e "Intervenções psicológicas durante a pandemia: possibilidades e desafios".

\section{Implicações na saúde mental em decorrência da pandemia do novo coronavírus}

Estudos sobre implicações na saúde mental em decorrência da pandemia do novo coronavírus ainda são escassos, por se tratar de fenômeno recente, mas apontam para repercussões negativas importantes. Além disso, pesquisas anteriores sobre outros surtos infecciosos revelaram desdobramentos desadaptativos, em curto, médio e longo prazo, para a população geral e para os profissionais da saúde (Jiang et al., 2020; Taylor, 2019). Por exemplo, na epidemia de Ebola de 1995, os sobreviventes relataram principalmente medo de morrer, de infectar outras pessoas, de se afastar ou sofrer abandono nas relações com familiares e amigos, bem como estigmatização social (Hall, Hall, \& Chapman, 2008). Os profissionais da saúde, por outro lado, reportaram sobretudo medo de contrair a doença e, ainda, transmiti-la a seus familiares, bem como sofrimento por estarem afastados de seus lares, estresse, sensação de perda de controle e de desvalorização, além de preocupação com o tempo de duração da epidemia (Hall et al., 2008)

Situação semelhante ocorreu em 2003, durante a epidemia de Síndrome Respiratória Aguda Grave (Severe Acute Respiratory Syndrome Coronavirus [SARS]), um outro tipo de coronavírus, quando as implicações psicológicas decorrentes da doença foram maiores que as implicações médicas, em termos de número de pessoas afetadas e tempo de duração em que elas foram afetadas (Taylor, 2019). No que diz respeito à COVID-19 em particular, os estudos desenvolvidos até o momento sobre as repercussões na saúde mental têm se voltado tanto à população geral quanto aos profissionais da saúde, destacando as particularidades desses dois grupos, tal como será abordado na presente seção.

\section{Saúde mental da população geral}

A rápida disseminação do novo coronavírus por todo o mundo, as incertezas sobre como controlar a doença e sobre sua gravidade, além da imprevisibilidade acerca do tempo de duração da pandemia e dos seus desdobramentos, caracterizam-se como fatores de risco à saúde mental da população geral (Zandifar \& Badrfam, 2020). Esse cenário parece agravado também pela difusão de mitos e informações equivocadas sobre a infecção e as medidas de prevenção, assim como pela dificuldade da população geral em compreender as orientações das autoridades sanitárias (Bao, Sun, Meng, Shi, \& Lu, 2020). Nesse sentido, videoclipes e mensagens alarmantes sobre a COVID-19 têm circulado em mídias sociais, por meio de smartphones e computadores, frequentemente provocando pânico (Goyal et al., 2020). Da mesma forma, notícias falsas vêm sendo compartilhadas (Barros-Delben et al., 2020; Shimizu, 2020), por vezes contrariando as orientações de autoridades sanitárias e minimizando os efeitos da doença. Isso parece contribuir para condutas inapropriadas e exposição a riscos desnecessários, pois os comportamentos que as pessoas apresentam estão ligados à compreensão que têm acerca da severidade da COVID-19 (Shojaei \& Masoumi, 2020).

Pessoas com suspeita de infecção pelo novo coronavírus podem desenvolver sintomas obsessivo-compulsivos, como a verificação repetida da temperatura corporal (Li et al., 2020b). A ansiedade em relação 
à saúde também pode provocar interpretação equivocada das sensações corporais, fazendo com que as pessoas as confundam com sinais da doença e se dirijam desnecessariamente a serviços hospitalares, conforme ocorreu na pandemia de influenza H1N1, em 2009 (Asmundson \& Taylor, 2020). Ademais, medidas como isolamento de casos suspeitos, fechamento de escolas e universidades, distanciamento social de idosos e outros grupos de risco, bem como quarentena, acabam por provocar diminuição das conexões face a face e das interações sociais rotineiras, o que também pode consistir em um estressor importante nesse período (Brooks et al., 2020; Zandifar \& Badrfam, 2020; Zhang, Wu, Zhao, \& Zhang, 2020b).

Dentre os estudos populacionais já realizados até o presente momento sobre implicações na saúde mental diante da pandemia do novo coronavírus, destaca-se o de Wang et al. (2020) com a população geral na China, incluindo 1.210 participantes em 194 cidades, durante o estágio inicial da pandemia. Esse estudo revelou sintomas moderados a severos de ansiedade, depressão e estresse, em $28,8 \%, 16,5 \%$ e $8,1 \%$ dos respondentes, respectivamente. Além disso, $75,2 \%$ dos respondentes referiram medo de que seus familiares contraíssem a doença. Ser mulher, estudante e apresentar sintomas físicos ligados à COVID-19, ou problemas de saúde prévios, foram fatores significativamente associados a maiores níveis de ansiedade, depressão e estresse. Por outro lado, receber informações precisas sobre a situação local da doença e formas de prevenção e tratamento consistiram em fatores significativamente associados a menores níveis de ansiedade, depressão e estresse (Wang et al., 2020).

A pandemia do novo coronavírus pode impactar a saúde mental e o bem-estar psicológico também devido a mudanças nas rotinas e nas relações familiares (Cluver et al., 2020; Ornell et al., 2020). Em 23 de março de 2020, o Fundo das Nações Unidas para a Infância divulgou que aproximadamente $95 \%$ das crianças e dos adolescentes matriculados nos sistemas de ensino da América Latina e do Caribe estavam temporariamente sem frequentar a escola em razão da COVID-19. A perspectiva era de que as escolas permanecessem fechadas por mais algumas semanas, podendo aumentar o risco de ocorrência de problemas de ensino-aprendizagem e evasão escolar, bem como reduzir o acesso à alimentação, água, práticas de higiene pessoal e programas recreacionais, sobretudo nas comunidades em situação de vulnerabilidade socioeconômica (Fundo das Nações Unidas para a Infância, 2020). Ademais, evidências têm revelado que há maior risco de crianças e adolescentes sofrerem violência quando escolas são fechadas devido a emergências de saúde (Rothe, Gallinetti, Lagaay, \& Campbell, 2015). Para mães, pais e demais cuidadores, o fato de estarem trabalhando remotamente ou mesmo impossibilitados de trabalhar, sem previsão sobre o tempo de duração dessa situação, tende a gerar estresse e medo, inclusive quanto às condições para a subsistência da família, reduzindo a capacidade de tolerância e aumentando o risco de violência contra crianças e adolescentes (Cluver et al., 2020).

Nota-se também o maior risco de violência contra mulheres nesse período, em que as vítimas costumam ficar confinadas junto aos agressores e, muitas vezes, não conseguem denunciar as agressões sofridas. Isso pareceu ocorrer em países como a China e a Itália durante a quarentena, conforme divulgado pela imprensa (Owen, 2020; Oliveira, 2020). Com base na experiência de outros países, algumas instituições no Brasil têm buscado ampliar os canais de denúncia durante o período de pandemia, por meio da disponibilização de comunicações online (via aplicativo de mensagens ou site), além de contato telefônico ou presencial nos órgãos da rede de enfrentamento a esse tipo de violência (Centro de Tecnologia da Informação e Comunicação do Estado do Rio Grande do Sul, 2020; Serviço de Rádio da Secretaria Executiva de Comunicação, 2020).

\section{Saúde mental dos profissionais da saúde}

Afora a população geral, profissionais da saúde também costumam experienciar estressores no contexto de pandemias, a saber: risco aumentado de ser infectado, adoecer e morrer; possibilidade de inadvertidamente infectar outras pessoas; sobrecarga e fadiga; exposição a mortes em larga escala; frustração 
por não conseguir salvar vidas, apesar dos esforços; ameaças e agressões propriamente ditas, perpetradas por pessoas que buscam atendimento e não podem ser acolhidas pela limitação de recursos; e afastamento da família e amigos (Taylor, 2019). Sobre a COVID-19 em particular, os desafios enfrentados pelos profissionais da saúde podem ser um gatilho para o desencadeamento ou a intensificação de sintomas de ansiedade, depressão e estresse (Bao et al., 2020), especialmente quando se trata daqueles que trabalham na chamada "linha de frente", ou seja, em contato direto com pessoas que foram infectadas pelo vírus (Li et al., 2020a). Em geral, esses profissionais vêm sendo desencorajados a interagir de maneira próxima com outras pessoas, o que tende a aumentar o sentimento de isolamento; têm lidado com mudanças frequentes nos protocolos de atendimento, em decorrência de novas descobertas sobre a COVID-19; e, ainda, costumam despender um tempo significativo do seu dia para colocar e remover os equipamentos de proteção individual, o que aumenta a exaustão relacionada ao trabalho (Zhang et al., 2020a). Nesse sentido, na China, equipes de saúde mental passaram a observar sinais de sofrimento psicológico, irritabilidade aumentada e recusa a momentos de descanso por parte de profissionais da saúde que trabalhavam na linha de frente (Chen et al., 2020).

Em estudo realizado por Zhang et al. (2020a) junto a 1.563 médicos que atuavam em hospitais de diferentes cidades chinesas, constatou-se a prevalência de sintomas de estresse em 73,4\% dos respondentes, depressão em 50,7\%, ansiedade em 44,7\%, e insônia em 36,1\%. No que diz respeito ao estresse e à insônia, em particular, é provável a ocorrência de um círculo vicioso, em que as dificuldades para dormir aumentavam os níveis de estresse e vice-versa (Zhang et al., 2020a). Outro estudo, realizado em um hospital chinês de grande porte, por meio de entrevistas a 13 médicos da linha de frente, revelou que esses profissionais demonstravam preocupação quanto à escassez de equipamentos de proteção, apresentavam dificuldades para lidar com pessoas que testaram positivo para o novo coronavírus e não compreendiam as recomendações ou se recusavam a aderir ao tratamento (ex.: quarentena no hospital), sentiam-se incapazes quando confrontados com casos graves, bem como temiam preocupar suas famílias e levar o vírus para suas casas (Chen et al., 2020).

Dentre as estratégias propostas para enfrentamento da pandemia do novo coronavírus, destaca-se o apelo para que a população geral fique em casa, buscando diminuir a transmissão, ao passo que a tendência é que os profissionais da saúde mantenham ou aumentem sua jornada de trabalho (Barros-Delben et al., 2020). Muitos profissionais da saúde que atuam na linha de frente, expostos ao vírus diariamente, foram infectados em todo o mundo; na Itália, esse número chegou a $20 \%$ no final do mês de março de 2020, de forma que o acesso a equipamentos de proteção individual para eles é preocupação central (The Lancet, 2020). No Brasil, a imprensa tem divulgado a escassez de equipamentos de proteção individual e o maior índice de licenças médicas a profissionais da saúde, como parece ser o caso de servidores municipais de São Paulo, na comparação entre a primeira e a segunda quinzena de março de 2020 (Rodrigues, 2020).

Ainda que não atuem na linha de frente ou que precisem se afastar dessa atuação temporariamente, profissionais da saúde podem apresentar sofrimento psicológico em contextos de emergências de saúde (Brooks et al., 2020; Li et al., 2020b). Nesse sentido, destaca-se o fenômeno da "traumatização vicária", também denominado "traumatização secundária", em que pessoas que não sofreram diretamente um trauma passam a apresentar sintomas psicológicos decorrentes da empatia por quem o sofreu (Li et al., 2020a). Em estudo realizado na China, Li et al. (2020a) investigaram a traumatização vicária relacionada à COVID-19 junto a uma amostra composta por 214 pessoas da população geral, 234 enfermeiros que trabalhavam na linha de frente e 292 enfermeiros que não trabalhavam na linha de frente $(n=740)$. Os achados evidenciaram níveis significativamente maiores de traumatização vicária em enfermeiros que não trabalhavam na linha de frente em comparação àqueles que trabalhavam na linha de frente. Segundo os autores, uma das possíveis explicações para esse resultado é que a traumatização vicária em enfermeiros que trabalham na linha de frente é derivada da empatia pelas pessoas que têm COVID-19, ao passo que enfermeiros que não trabalham na linha de frente mostram empatia pelas pessoas que têm COVID-19, mas também empatia e 
preocupação com os colegas da linha de frente. Além disso, enfermeiros que trabalham na linha de frente podem ter maior preparo e habilidades psicológicas para lidar com emergências de saúde em comparação àqueles que não trabalham na linha de frente (Li et al., 2020b). Assim, mesmo quando precisam se afastar das funções laborais (ex.: quando a quarentena é necessária), profissionais da saúde tendem a reportar culpa, raiva, frustração e tristeza (Brooks et al., 2020), o que sugere a importância da atenção psicológica a essa população no contexto de pandemias.

\section{Intervenções psicológicas durante a pandemia: possibilidades e desafios}

Intervenções psicológicas voltadas tanto à população geral quanto aos profissionais da saúde desempenham um papel central para lidar com as implicações na saúde mental em decorrência da pandemia do novo coronavírus (Bao et al., 2020; Shojaei \& Masoumi, 2020; Zhou, 2020). Autoridades sanitárias, organizações ligadas à saúde e cientistas em diferentes países têm divulgado orientações para práticas alinhadas às demandas do atual contexto (American Psychological Association, 2020; CFP, 2020a; Jung \& Jun, 2020; WHO, 2020b). Em geral, recomenda-se que as intervenções psicológicas face a face sejam restritas ao mínimo possível (ex.: atendimento a profissionais da saúde que trabalham na linha de frente e não foram infectados), para minimizar o risco de propagação do vírus (Jiang et al., 2020). Assim, têm sido sugeridos serviços psicológicos realizados por meios de tecnologia da informação e da comunicação, incluindo Internet, telefone e carta (Jiang et al., 2020; Wang et al., 2020; Xiao, 2020). Em outras epidemias, como a SARS, o atendimento psicológico remoto tornou-se rapidamente um mecanismo importante para acolhimento a queixas relativas à saúde mental (Duan \& Zhu, 2020).

No Brasil, em 26 de março de 2020, foi publicada a Resolução CFP n 4/2020, que permite a prestação de serviços psicológicos por meios de tecnologia da informação e da comunicação após realização do "Cadastro e-Psi", embora não seja necessário aguardar a emissão de parecer para iniciar o trabalho remoto. A Resolução CFP n ${ }^{\circ}$ 4/2020 suspende, durante o período de pandemia do novo coronavírus, os Art. $3^{\circ}, 4^{\circ}, 6^{\circ}$, $7^{\circ}$ e $8^{\circ}$ da Resolução CFP n 11/2018. Portanto, passa a ser autorizada a prestação de serviços psicológicos por meios de tecnologia da informação e da comunicação a pessoas e grupos em situação de urgência, emergência e desastre, bem como de violação de direitos ou violência, buscando minimizar as implicações psicológicas diante da COVID-19 (CFP, 2020a).

Dadas a crescente demanda relacionada à saúde mental nesse período, a escassez de profissionais capacitados para acolhê-la, e a necessidade de respostas rápidas e eficientes, algumas localidades têm proposto uma classificação de pessoas e grupos afetados pelo novo coronanírus, a ser considerada na priorização para a oferta das intervenções. A Comissão Nacional de Saúde da China, por exemplo, propôs uma classificação em quatro níveis: (1) Casos mais vulneráveis a problemas de saúde mental, como pessoas hospitalizadas com infecção confirmada e profissionais da saúde que trabalhem ou não na linha de frente; (2) Pessoas isoladas com sintomas leves, suspeitas de infecção ou em contato próximo com casos confirmados; pessoas com sintomas como febre; (3) Pessoas em contato próximo com casos descritos nos níveis 1 e 2, ou seja, familiares, amigos e colegas; equipes de resgate que participem de ações de resposta à COVID-19; (4) Pessoas afetadas pelas medidas de prevenção e controle, grupos suscetíveis e população geral (Duan \& Zhu, 2020; Jiang et al., 2020; Li et al., 2020b). Sistemas semelhantes de classificação também foram propostos na Coreia do Sul (Jung \& Jun, 2020) e no Irã (Shojaei \& Masoumi, 2020). Considerando as recomendações de priorização tanto da população geral quanto dos profissionais da saúde para as intervenções psicológicas no atual contexto de pandemia, serão apresentados, a seguir, possibilidades e desafios da prática junto a cada um desses grupos. 


\section{Intervenções voltadas à população geral}

As intervenções voltadas à população geral incluem, por exemplo, propostas psicoeducativas, tais como: cartilhas e outros materiais informativos (Wang et al., 2020; Weide, Vicentini, Araujo, Machado, \& Enumo, 2020); oferta de canais para escuta psicológica, de modo que as pessoas possam aliviar suas emoções negativas via ligação telefônica ou atendimento em plataformas online, 24 horas por dia e sete dias por semana (Jiang et al., 2020; Zhou, 2020); atendimentos psicológicos por meio de cartas estruturadas, em que inicialmente o usuário do serviço se apresenta e descreve suas principais emoções e queixas, além das possíveis razões para elas (Xiao, 2020); atendimentos psicológicos online (Duan \& Zhu, 2020; Li et al., 2020b) ou, quando comprovadamente necessários, presenciais (CFP, 2020b; Jiang et al., 2020). Levantamentos online também têm sido realizados para melhor compreender o estado de saúde mental da população diante da COVID-19, com o objetivo de identificar rapidamente casos com maior risco e ofertar intervenções psicológicas alinhadas às demandas (Zhou, 2020).

Nesse contexto, ainda que de forma remota, sugere-se inicialmente a oferta de primeiros cuidados psicológicos, os quais envolvem assistência humana e ajuda prática em situações de crise, buscando aliviar preocupações, oferecer conforto, ativar a rede de apoio social e suprir necessidades básicas (ex.: água, alimentação e informação) (WHO, 2011). Ademais, as intervenções psicológicas devem ser dinâmicas e, primeiramente, focadas nos estressores relacionados à doença ou nas dificuldades de adaptação às restrições do período (Zhang et al., 2020b). Sobre as temáticas que vêm sendo abordadas pelos profissionais da saúde mental junto à população geral, destacam-se: informações sobre reações esperadas no contexto de pandemia, como sintomas de ansiedade e estresse, além de emoções negativas, como tristeza, medo, solidão e raiva (Weide et al., 2020); estratégias para promoção de bem-estar psicológico, a exemplo de medidas para organização da rotina de atividades diárias sob condições seguras, cuidado com o sono, prática de atividades físicas e técnicas de relaxamento (Banerjee, 2020); fortalecimento das conexões com a rede de apoio social, ainda que os contatos não ocorram face a face, considerando que instituições como escolas, empresas e igrejas costumam estar fechadas, o que pode gerar sentimentos de solidão e vulnerabilidade (Shojaei \& Masoumi, 2020); cuidado com a exposição excessiva a informações, incluindo noticiários na televisão e em outras mídias (Barros-Delben et al., 2020); e importância da checagem da veracidade das informações (Bao et al., 2020).

Para pessoas que experienciam níveis de sofrimento mais severos relacionados à pandemia, intervenções psicológicas mais intensivas tendem a ser necessárias (Taylor, 2019). Esses casos frequentemente incluem pessoas com suspeita ou diagnóstico confirmado e seus familiares (Shojaei \& Masoumi, 2020), pessoas hospitalizadas ou que passaram pela experiência de hospitalização (Duan \& Zhu, 2020), pessoas que estão vivenciando o processo de terminalidade ou a morte de familiares (Li et al., 2020b), em particular aquelas que não puderam se despedir presencialmente ou acompanhar o falecido em razão da pandemia (Barros-Delben et al., 2020). As demandas psicológicas tendem a se modificar de acordo com a progressão da doença ou a ocorrência de fatos relacionados a ela, o que se alinha a intervenções psicológicas dinâmicas (Zhang et al., 2020b). Sempre que necessário, devem-se fazer encaminhamentos a outros profissionais ou serviços de saúde (Taylor, 2019).

Dentre os possíveis desafios para o trabalho de psicólogos na vigência da pandemia de COVID-19 no Brasil, destacam-se a restrição a deslocamentos e a necessidade de realização de serviços psicológicos predominantemente por meios de tecnologia da informação e da comunicação. Em primeiro lugar, muitos brasileiros não têm acesso à Internet, o que limita a possibilidade de oferta de apoio nesse momento. Ademais, ainda que tenham acesso à Internet, algumas pessoas podem apresentar dificuldades para utilizar smartphones ou computadores. Isso tende a acontecer com idosos, faixa etária em que tem ocorrido o maior número de complicações e óbitos decorrentes da COVID-19 (Ornell et al., 2020). Portanto, nesses casos, sugere-se a realização de serviços psicológicos via telefone (Li et al., 2020b; Shojaei \& Masoumi, 2020). Nesse sentido, 
em 23 de março de 2020, o CFP enviou um ofício circular a gestores públicos, empregadores de psicólogos e usuários de serviços. Por meio desse documento, recomendou-se a suspensão das atividades de psicólogos na modalidade presencial em todo o país, com exceção daquelas comprovadamente emergenciais, ocasião em que devem ser ofertadas condições adequadas de prevenção e proteção contra o novo coronavírus, incluindo máscaras e álcool 70\% (CFP, 2020b).

No que diz respeito às cartilhas e aos materiais informativos, é importante que sejam elaborados em linguagem acessível, com diagramação visualmente atrativa e ajustada às características do público-alvo. Considerar esses aspectos é fundamental, sobretudo porque pessoas com menor nível de escolaridade costumam apresentar maior dificuldade para compreender informações relacionadas à saúde (Shojaei \& Masoumi, 2020), bem como maior tendência a manifestar sintomas psicológicos em decorrência da pandemia do novo coronavírus (Wang et al., 2020). Assim, no Brasil, materiais informativos disponibilizados em áudio e vídeo (i.e., que não demandem leitura) podem ser uma boa alternativa, especialmente para pessoas com menor nível de escolaridade. Sugere-se ainda a confecção de materiais informativos para populações específicas, como pessoas que têm COVID-19, seus familiares, casos suspeitos e pessoas que apresentem problemas de saúde mental preexistentes (Zhou, 2020). Psicólogos também podem contribuir em iniciativas para combate ao estigma relacionado à COVID-19, desmistificando a ideia de que a doença seria vinculada a uma nacionalidade específica (WHO, 2020b), o que tem levado à xenofobia (Shimizu, 2020) e, ainda, incentivando a utilização de termos como "pessoas que têm COVID-19" ou "pessoas em recuperação de COVID-19", em substituição a termos como "doentes", "vítimas" ou "famílias COVID-19" (WHO, 2020b).

\section{Intervenções voltadas aos profissionais da saúde}

Dadas as rigorosas medidas que os serviços de saúde adotam para contenção da infecção, o contato direto entre o psicólogo e as pessoas que têm COVID-19 costuma ser raro (Jiang et al., 2020). Assim, profissionais da saúde que trabalham na linha de frente, como enfermeiros e médicos, serão aqueles que predominantemente escutarão queixas e oferecerão apoio psicológico às pessoas que buscam os serviços de saúde ou que estão hospitalizadas (Duan \& Zhu, 2020). Portanto, psicólogos podem contribuir para promoção da saúde mental e prevenção de implicações psicológicas negativas a profissionais da saúde, ao oferecer a eles suporte e orientação sobre como manejar algumas situações. Isso parece importante, pois, dentre os desafios por eles relatados, destaca-se atender pessoas que testaram positivo para o novo coronavírus e que não compreendem as recomendações ou se recusam a aderir ao tratamento (Chen et al., 2020), bem como lidar com a frustração por não conseguir salvar vidas, apesar de todos os esforços (Taylor, 2019).

Além disso, muitos profissionais da saúde no Brasil não têm experiência de atuação em emergências de grande porte, como é o caso da COVID-19, o que representa um estressor adicional (Barros-Delben et al., 2020). Logo, sugere-se a realização de intervenções voltadas à orientação sobre sintomas psicológicos que profissionais da saúde podem apresentar nesse contexto (ex.: estresse, depressão, ansiedade e insônia; Zhang et al., 2020a), bem como estratégias de enfrentamento e autocuidado (ex.: gerenciamento de estresse e importância dos momentos de descanso; Taylor, 2019). Adicionalmente, considerando relatos de profissionais da saúde sobre preocupações e sentimento de isolamento pelo afastamento da família e dos amigos (Taylor, 2019), psicólogos podem contribuir para o fortalecimento da rede de apoio, ao incentivá-los a manter contatos frequentes, durante os intervalos no trabalho, por meio de telefonemas, mensagens de texto, áudio e vídeo (Chen et al., 2020). Isso tende a beneficiar também a saúde mental das pessoas da rede de apoio dos profissionais da saúde, pois mantê-las informadas pode reduzir as emoções negativas, como o medo (Banerjee, 2020).

As intervenções psicológicas junto aos profissionais da saúde podem ocorrer em plataformas online, por ligações telefônicas (Chen et al., 2020; Li et al., 2020b), face a face, se necessário (Jiang et al., 2020), 
ou, ainda, com cartilhas e outros materiais informativos (Zhou, 2020). Frente aos indicativos de implicações psicológicas negativas a profissionais da saúde diante da pandemia do novo coronavírus (Zhang et al., 2020a), é importante que essas intervenções sejam precoces (Banerjee, 2020) e que incluam também aqueles que não estão na linha de frente, os quais podem reportar culpa, raiva, frustração e tristeza (Brooks et al., 2020), além de traumatização vicária (Li et al., 2020a).

Sobre os desafios para a atuação do psicólogo junto aos profissionais da saúde, destaca-se a possibilidade de baixa adesão às intervenções, em função da falta de tempo e do cansaço pela sobrecarga de trabalho, em particular para aqueles que estão na linha de frente (Li et al., 2020b). Ademais, no Brasil, é possível que profissionais da saúde se preocupem com questões como escassez de equipamentos de proteção individual, e venham a considerar as intervenções psicológicas como secundárias. Assim, para psicólogos que atuam em hospitais e outros serviços de saúde, sugere-se a realização de visitas à área de descanso para escutar os desafios vivenciados pelos profissionais e acolhê-los (Chen et al., 2020), ou mesmo para sensibilizá-los ou estimulá-los a buscar auxílio psicológico, se necessário.

\section{Considerações Finais}

No presente estudo, foram sistematizados conhecimentos sobre implicações na saúde mental e intervenções psicológicas diante da pandemia do novo coronavírus. Em suma, compreende-se que a Psicologia pode oferecer contribuições importantes para o enfrentamento das repercussões da COVID-19, que vem sendo considerada a maior emergência de saúde pública que a comunidade internacional enfrenta em décadas. Essas contribuições envolvem a realização de intervenções psicológicas durante a vigência da pandemia para minimizar implicações negativas e promover a saúde mental, bem como em momentos posteriores, quando as pessoas precisarão se readaptar e lidar com as perdas e transformações.

Este estudo consistiu em uma revisão narrativa da literatura, de modo que pesquisas adicionais sobre a temática são essenciais. Nesse sentido, sugerem-se levantamentos sobre implicações na saúde mental diante da pandemia e sobre intervenções psicológicas alinhadas às necessidades do contexto brasileiro, considerando as características de diferentes populações atingidas pela COVID-19 e, em particular, de pessoas e grupos em maior vulnerabilidade socioeconômica. Entende-se que, embora imponha desafios adicionais à atuação dos psicólogos no Brasil e no mundo, a pandemia do novo coronavírus pode contribuir para o aperfeiçoamento da prática e da pesquisa em situações de crise, emergência e desastre.

\section{Contribuição}

B. SCHMIDT, M. A. CREPALDI e S. D. A. BOLZE contribuíram com a concepção e desenho, análise, discussão dos resultados, revisão e aprovação da versão final do artigo. L. NEIVA-SILVA e L. M. DEMENECH contribuíram com análise e discussão dos resultados, revisão e aprovação da versão final do artigo.

Referências
American Psychological Association. (2020). Pandemics. Retrieved from https://www.apa.org/practice/programs/dmhi/
research-information/pandemics
Asmundson, G. J. G., \& Taylor, S. (2020). Coronaphobia: fear and the 2019-nCoV outbreak. Journal of Anxiety Disorders,
70, 102196. http://dx.doi.org/10.1016/j.janxdis.2020.102196
Banerjee, D. (2020). The COVID-19 outbreak: crucial role the psychiatrists can play. Asian Journal of Psychiatry, 50,
102014. http://dx.doi.org/10.1016/j.ajp.2020.102014


Bao, Y., Sun, Y., Meng, S., Shi, J., \& Lu, L. (2020). 2019-nCoV epidemic: address mental health care to empower society. The Lancet, 395(10224), e37-e38. http://dx.doi.org/10.1016/S0140-6736(20)30309-3

Barros-Delben, P., Cruz, R. M., Trevisan, K. R. R., Gai, M. J. P., Carvalho, R. V. C., Carlotto, R. A. C., ... MalloyDiniz, L. F. (2020). Saúde mental em situação de emergência: COVID-19 [Ahead of print]. Revista Debates in Psychiatry, 10, 2-12. Recuperado de https://d494f813-3c95-463a-898c-ea1519530871.filesusr.com/ugd/c37608_ e2757d5503104506b30e50caa 6fa6aa7.pdf

Brooks, S. K., Webster, R. K., Smith, L. E., Woodland, L., Wessely, S., Greenberg, N., \& Rubin, G. J. (2020). The psychological impact of quarantine and how to reduce it: rapid review of the evidence. The Lancet, 395(10227), 912-920. http:// dx.doi.org/10.1016/S0140-6736(20)30460-8

Carvalho, P. M. M., Moreira, M. M., Oliveira, M. N. A., Landim, J. M. M., \& Rolim Neto, M. L. (2020). The psychiatric impact of the novel coronavirus outbreak. Psychiatry Research, 286(112902), 1-2. http://dx.doi.org/10.1016/j. psychres.2020.112902

Centro de Tecnologia da Informação e Comunicação do Estado do Rio Grande do Sul. (2020). Delegacia Online amplia possibilidades de registro de ocorrência. Recuperado de https://www.procergs.rs.gov.br/delegacia-online-ampliapossibilidades-de-registro-de-ocorrencia

Chen, Q., Liang, M., Li, Y., Guo, J., Fei, D., Wang, L., ... Zhang, Z. (2020). Mental health care for medical staff in China during the COVID-19 outbreak. The Lancet, 7(4), 15-16. http://dx.doi.org/10.1016/S2215-0366(20)30078-X

Cluver, L., Lachman, J. M., Sherr, L., Wessels, I., Krug, E., Rakotomalala, S., ... McDonald, K. (2020). Parenting in a time of COVID-19. The Lancet, 395, e64. http://dx.doi.org/10.1016/S0140-6736(20)30736-4

Consejo General de la Psicología de España. (2020, 3 de octubre). Recursos de ayuda psicológica para afrontar el Covid-19. Recuperado el http://www.infocop.es/view_article.asp?id=8670\&cat=44

Conselho Federal de Psicologia. (2020a). Resolução do exercício profissional n4, de 26 de março de 2020. Dispõe sobre regulamentação de serviços psicológico prestados por meio de Tecnologia da Informação e da Comunicação durante a pandemia do COVID19. Recuperado de https://atosoficiais.com.br/cfp/resolucao-do-exercicio-profissional-n-42020-dispoe-sobre-regulamentacao-de-servicos-psicologicos-prestados-por-meio-de-tecnologia-da-informacao-e-dacomunicacao-durante-a-pandemia-do-covid19? origin=instituicao

Conselho Federal de Psicologia. (2020b). Ofício-Circular n 40/2020/GTec/CG-CFP. Recuperado de https://site.cfp.org. br/wp-content/uploads/2020/03/SEI_CFP-0214041-Of\%C3\%ADcio-Circular_.pdf

Duan, L., \& Zhu, G. (2020). Psychological interventions for people affected by the COVID-19 epidemic. The Lancet Psychiatry, 7(4), 300-302. http://dx.doi.org/10.1016/\$2215-0366(20)30073-0

Ferguson, N., Laydon, D., Nedjati Gilani, G., Imai, N., Ainslie, K., Baguelin, M., ... Ghani, A. (2020). Report 9: impact of non-pharmaceutical interventions (NPIS) to reduce COVID19 mortality and healthcare demand. http://dx.doi. org/10.25561/77482

Fundo das Nações Unidas para a Infância. (2020). COVID-19: More than 95 per cent of children are out of school in Latin America and the Caribbean. Retrieved from https://www.unicef.org/press-releases/covid-19-more-95-cent-childrenare-out-school-latin-america-and-caribbean

Goyal, K., Chauhan, P., Chhikara, K., Gupta, P., \& Singh, M. P. (2020). Fear of COVID 2019: first suicidal case in India. Asian Journal of Psychiatry, 49(101989). http://dx.doi.org/10.1016/j.ajp.2020.101989

Hall, R. C. W., Hall, R., \& Chapman, M. J. (2008). The 1995 Kikwit Ebola outbreak: lessons hospitals and physicians can apply to future viral epidemics. General Hospital Psychiatry, 30(5), 446-452. http://dx.doi.org/10.1016/j. genhosppsych.2008.05.003

Jiang, X., Deng, L., Zhu, Y., Ji, H., Tao, L., Liu, L., ... Ji, W. (2020). Psychological crisis intervention during the outbreak period of new coronavirus pneumonia from experience in Shanghai. Psychiatry Research, 286, 112903. http://dx.doi. org/10.1016/j.psychres.2020.112903

Jung, S. J., \& Jun, J. Y. (2020). Mental health and psychological intervention amid COVID-19 Outbreak: perspectives from South Korea. Yonsei Medical Journal, 61(4), 271-272. http://dx.doi.org/10.3349/ymj.2020.61.4.271

Li, Z., Ge, J., Yang, M., Feng, J., Qiao, M., Jiang, R., ... Yang, C. (2020a). Vicarious traumatization in the general public, members, and non-members of medical teams aiding in COVID-19 control. Brain, Behavior, and Immunity. http:// dx.doi.org/10.1016/j.bbi.2020.03.007

Li, W., Yang, Y., Liu, Z. H., Zhao, Y. J., Zhang, Q., Zhang, L., ... Xiang, Y. T. (2020b). Progression of mental health services during the COVID-19 outbreak in China. International Journal of Biological Sciences, 16(10), 1732-1738. http://dx.doi. org/10.7150/ijbs.45120 
Matos, H. J. (2018). A próxima pandemia: estamos preparados? Revista Pan-Amazônica de Saúde, 9(3), 9-11. http:// dx.doi.org/10.5123/S2176-62232018000300001

Ministério da Saúde. (2020a). Painel de casos de doença pelo coronavírus 2019 (COVID-19). Recuperado de https:// covid.saude.gov.br/

Ministério da Saúde. (2020b). Portaria n 639, de 31 de março de 2020. Dispõe sobre a Ação Estratégica "O Brasil Conta Comigo - Profissionais da Saúde", voltada à capacitação e ao cadastramento de profissionais da área de saúde, para o enfrentamento à pandemia do coronavírus (COVID-19). Diário Oficial da União. Brasília: Autor. Recuperado de http:// www.in.gov.br/en/web/dou/-/portaria-n-639-de-31-de-marco-de-2020-250847738

Oliveira, M. (2020, 27 de março). Em quarentena total, mulheres não conseguem denunciar violência doméstica na Itália. Folha de S. Paulo. Recuperado de https://www1.folha.uol.com.br/mundo/2020/03/em-quarentena-total-mulheresnao-conseguem-denunciar-violencia-domestica-na-italia.shtml

Ornell, F., Schuch, J. B., Sordi, A. O., \& Kessler, F. H. P. (2020). "Pandemic fear" and COVID-19: mental health burden and strategies. Brazilian Journal of Psychiatry. Retrieved from https://www.rbppsychiatry.org.br/details/943/en-US/pandemic-fear--and-covid-19--mental-health-burden-and-strategies

Owen, L. (2020, March 8). Coronavirus: five ways virus upheaval is hitting women in Asia. BBC News. Retrieved from https://www.bbc.com/news/world-asia-51705199

Pautasso, M. (2020). The structure and conduct of a narrative literature review. In R. S. Tubbs, S. M. Buerger, M. M. Shoja, A. Arynchyna \& M. Karl (Eds.), A guide to the scientific career: virtues, communication, research, and academic writing (pp.299-310). Hoboken: Wiley Blackwell.

Rodrigues, A. (2020, 31 de março). Afastamentos por suspeitas de coronavírus explodem entre profissionais da saúde. Folha de S. Paulo. Recuperado de https://www1.folha.uol.com.br/equilibrioesaude/2020/03/com-alta-do-coronaviruslicencas-medicas-de-servidores-da-saude-aumentam-57-em-sp.shtml

Rothe, D., Gallinetti, J., Lagaay, M., \& Campbell, L. (2015). Ebola: beyond the health emergency. Retrieved from https:// plan-international.org/publications/ebola-beyond-health\%C2\%A0emergency

Russell, T. W., Hellewell, J., Abbott, S., Jarvis, C. I., van Zandvoort, K., CMMID nCov working group, Flasche, S., ... Kucharski, A. J. (2020). Using a delay-adjusted case fatality ratio to estimate under-reporting. Retrieved from https:// cmmid.github.io/topics/covid19/severity/global_cfr_estimates.html

Serviço de Rádio da Secretaria Executiva de Comunicação. (Produtor). (2020, 25 de março). Coronavírus em SC: Polícia Civil intensifica proteção e canais de denúncia contra violência contra mulheres [Áudio podcast]. Recuperado de https://www.sc.gov.br/noticias/radio/coronavirus-em-sc-policia-civil-intensifica-protecao-e-canais-de-denuncia-contraviolencia-contra-mulheres

Schmidt, B., Crepaldi, M. A., Bolze, S. D. A., Neiva-Silva, L., \& Demenech, L. M. (2020). Impactos na Saúde Mental e Intervenções Psicológicas Diante da Pandemia do Novo Coronavírus (COVID-19). Scielo Preprints. Versão 1. http:// dx.doi.org/10.1590/SciELOPreprints.58

Shimizu, K. (2020). 2019-nCoV, fake news, and racism. The Lancet, 395(10225), 685-686. http://dx.doi.org/10.1016/ S0140-6736(20)30357-3

Shojaei, S. F., \& Masoumi, R. (2020). The importance of mental health training for psychologists in COVID-19 outbreak. Middle East Journal of Rehabilitation and Health Studies, 7(2), e102846. http://dx.doi.org/10.5812/mejrh.102846

Taylor, S. (2019). The psychology of pandemics: preparing for the next global outbreak of infectious disease. Newcastle upon Tyne: Cambridge Scholars Publishing.

The Lancet (2020). COVID-19: protecting health-care workers (Editorial). The Lancet, 395(10228), 922. http://dx.doi. org/10.1016/S0140-6736(20)30644-9

Villela, D. A. M. (2020). The value of mitigating epidemic peaks of COVID-19 for more effective public health responses. Revista da Sociedade Brasileira de Medicina Tropical, 53, e20200135. http://dx.doi.org/10.1590/0037-8682-0135-2020

Weide, J. N., Vicentini, E. C. C., Araujo, M. F., Machado,W. L., \& Enumo, S. R. F. (2020). Cartilha para enfrentamento do estresse em tempos de pandemia. Porto Alegre: PUCRS/PUC-Campinas.

World Health Organization. (2011). Psychological first aid: Guide for field workers. Geneva: Author. Retrieved from https://www.who.int/mental_health/publications/guide_field_workers/en/ 
World Health Organization. (2020a). Coronavirus disease (COVID-19) situation dashboard. Geneva: Author. Retrieved from https://covid19.who.int/

World Health Organization. (2020b). Mental health and psychosocial considerations during the COVID-19 outbreak. Geneva: Author. Retrieved from https://www.who.int/docs/default-source/coronaviruse/mental-health-considerations.pdf

Xiao, C. (2020). A novel approach of consultation on 2019 novel coronavirus (COVID-19)-related psychological and mental problems: structured letter therapy. Psychiatry Investigation, 17(2), 175-176. http://dx.doi.org/10.30773/pi.2020.0047

Zandifar, A., \& Badrfam, R. (2020). Iranian mental health during the COVID-19 epidemic. Asian Journal of Psychiatry, 51, 101990. http://dx.doi.org/10.1016/j.ajp.2020.101990

Zhang, C., Yang, L., Liu, S., Ma, S., Wang, Y., Cai, Z., ... Zhang, B. (2020a). Survey of insomnia and related social psychological factors among medical staff involved in the 2019 novel coronavirus disease outbreak. Frontiers in Psychiatry, 11(306), 1-9. http://dx.doi.org/10.3389/fpsyt.2020.00306

Zhang, J., Wu, W., Zhao, X., \& Zhang, W. (2020b). Recommended psychological crisis intervention response to the 2019 novel coronavirus pneumonia outbreak in China: a model of West China Hospital. Precision Clinical Medicine, 3(1), 3-8. http://dx.doi.org/10.1093/pcmedi/pbaa006

Zhou, X. (2020). Psychological crisis interventions in Sichuan Province during the 2019 novel coronavirus outbreak. Psychiatry Research, 286, 112895. http://dx.doi.org/10.1016/j.psychres.2020.112895

Recebido em: abril 7,2020

Versão Final: abril 17,2020

Aprovado: abril 17,2020 